International Journal of Pure and Applied Mathematics

Volume 113 No. 1 2017, 59-71

ISSN: 1311-8080 (printed version); ISSN: 1314-3395 (on-line version)

url: http://www.ijpam.eu

doi: $10.12732 /$ ijpam.v113i1.7

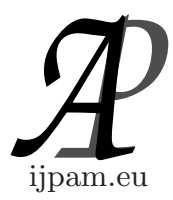

\title{
ASYMPTOTICALLY $\omega$-PERIODIC SOLUTION FOR AN EVOLUTION DIFFERENTIAL EQUATION VIA $\omega$-PERIODIC LIMIT FUNCTIONS
}

\author{
William Dimbour ${ }^{1}$, Solym Mawaki ${ }^{2}$ \\ ${ }^{1}$ UMR Espace-Dev \\ Université de Guyane \\ Campus de Troubiran 97300 \\ Cayenne Guyane (FWI), FRENCH GUIANA \\ ${ }^{2}$ Centre Universitaire de Mayotte \\ Département Sciences et Technologies \\ Route Nationale 3, BP 53 - 97660, DEMBENI
}

\begin{abstract}
In this paper, we give sufficient conditions for the existence and uniqueness of asymptotically $\boldsymbol{\omega}$-periodic solutions for an evolution differential equation considering the class of $\boldsymbol{\omega}$-periodic limit functions. This is done using the Banach Fixed Point Theorem.
\end{abstract}

AMS Subject Classification: $34 \mathrm{~K} 05,34 \mathrm{~A} 12,34 \mathrm{~A} 40$

Key Words: asymptotically $\omega$-periodic functions, evolutionnary process, oridnary differential equation

\section{Introduction}

The existence of periodic solutions and its various extensions to abstract Cauchy problem has been studied in Several works([6]-[9]). Recently, the concept of $\omega$ periodic limit functions has been introduced by Xie and Zhang in [10]. The class of $\omega$-periodic limit functions generalize asymptotically $\omega$-periodic func-

Received: October 14, 2016

Revised: January 17, 2017

Published: February 28, 2017

$\S_{\text {Correspondence author }}$ (c) 2017 Academic Publications, Ltd. url: www.acadpubl.eu 
tions $([11],[12])$ in a different way from $S$-asymptotically $\omega$-periodic functions ([1]-[5]) and have some relationship with asymptotically $\omega$-periodic functions in the Stepanov sense $([6],[9])$. The concept of $S$-asymptotically $\omega$-periodic have many applications in functional differential equations, integro-differential equations, fractionnal differential equation. In [10], Xie and Zhang investigate some properties of $\omega$-periodic limit functions in order to study the existence and uniqueness of asymptotically $\omega$-periodic mild solutions of the following abstract Cauchy problem

$$
\left\{\begin{array}{l}
x^{\prime}(t)=A x(t)+f(t, x(t)) d t, \\
x(0)=c_{0},
\end{array}\right.
$$

where $A$ is the infinitesimal generator of an exponentially stable $C_{0}$-semigroup $(T(t))_{t \geq 0}$. The aim of this work is to investigate properties of $\omega$-periodic limit functions and to study the existence of asymptotically $\omega$-periodic mild solution to the semilinear differential equation.

$$
\left\{\begin{array}{l}
x^{\prime}(t)=A(t) x(t)+f(t, x(t)) d t \\
x(0)=c_{0}
\end{array}\right.
$$

where $c_{0} \in \mathbb{X}$, and $A(t)$ generates an exponentially stable $\omega$-perioic evolutionary family in $\mathbb{X}$. The paper is organised as follows. We recall in section 2 , definitions and properties of $\omega$-periodic limit functions. In section 2 , we present also new properties of $\omega$-periodic functions. In section 3 , we study the existence and uniqueness of asymptotically $\omega$-periodic solution of the equation (1). This result is obtained, using the Banach fixed point theorem.

\section{Preliminaries}

Let $\mathcal{C}_{b}\left(\mathbb{R}^{+}, \mathbb{X}\right)$ the space consisting of bounded and continuous functions from $\mathbb{R}^{+}$into $\mathbb{X}$, endowed with the uniform convergence norm $\|\cdot\|_{\infty}$. Set $C_{0}\left(\mathbb{R}^{+}, \mathbb{X}\right)=$ $\left\{f \in \mathcal{C}_{b}\left(\mathbb{R}^{+}, \mathbb{X}\right)=\lim _{t \rightarrow \infty}\|f(t)\|=0\right\}$ and $P_{\omega}\left(\mathbb{R}^{+}, \mathbb{X}\right)=\left\{f \in \mathcal{C}_{b}\left(\mathbb{R}^{+}, \mathbb{X}\right)\right.$ : $f$ is $\omega-$ periodic $\}$.

Definition 2.1. A function $f \in \mathcal{C}_{b}\left(\mathbb{R}^{+}, \mathbb{X}\right)$ is said to be asymptotically $\omega$-periodic if it can be expressed as $f=g+h$, where $g \in P_{\omega}\left(\mathbb{R}^{+}, \mathbb{X}\right)$ and $h \in C_{0}\left(\mathbb{R}^{+}, \mathbb{X}\right)$. The subspace of $\mathcal{C}_{b}\left(\mathbb{R}^{+}, \mathbb{X}\right)$ consisting of the asymptotically $\omega$-periodic functions will be denoted by $A P_{\omega}\left(\mathbb{R}^{+}, \mathbb{X}\right)$.

Definition 2.2. Let $f \in \mathcal{C}_{b}\left(\mathbb{R}^{+}, \mathbb{X}\right)$ and $\omega>0$. We call $f \omega$-periodic limit if $g(t)=\lim _{n \rightarrow \infty} f(t+n \omega)$ is well defined for each $t \in \mathbb{R}^{+}$, where $n \in \mathbb{N}$. The collection of such functions will be denoted by $P_{\omega} L\left(\mathbb{R}^{+}, \mathbb{X}\right)$. 
Remark 2.1. The function $g$ is measurable but not necessarily continuous.

Proposition 2.1. (see [10]) If $f, f_{1}$ and $f_{2}$ are $\omega$-periodic limit and $g(t)=\lim _{n \rightarrow \infty} f(t+n \omega)$ is well defined for each $t \in \mathbb{R}^{+}$, then the following statements are true:

(1) $f_{1}+f_{2}$ is $\omega$-periodic limit;

(2) $c f$ is $\omega$-periodic limit for every scalac $c$;

(3) $g(t+\omega)=g(t)$ for each $t \in \mathbb{R}^{+}$;

(4) $g$ is bounded on $\mathbb{R}^{+}$; moreover $\|g\|_{\infty} \leq\|f\|_{\infty}$;

(5) $f_{a}(t)=f(t+a)$ is $\omega$-periodic limit for each fixed $a \in \mathbb{R}^{+}$.

Theorem 2.2. (see [10]) $P_{\omega} L\left(\mathbb{R}^{+}, \mathbb{X}\right)$ is a Banach space.

Proposition 2.3. (see [10]) Let $f \in P_{\omega} L\left(\mathbb{R}^{+}, \mathbb{X}\right)$ and $g(t)=\lim _{n \rightarrow \infty} f(t+$ $n \omega)$ be well defined for each $t \in \mathbb{R}^{+}$. If $g(t)=\lim _{n \rightarrow \infty} f(t+n \omega)$ uniformly on $[0, \omega]$, then $f \in A P_{\omega}\left(\mathbb{R}^{+}, \mathbb{X}\right)$.

Corollary 2.4. (see [10]) Let $f \in P_{\omega} L\left(\mathbb{R}^{+}, \mathbb{X}\right)$ and $g(t)=\lim _{n \rightarrow \infty} f(t+$ $n \omega)$ be well defined for each $t \in \mathbb{R}^{+}$. If $g(t)=\lim _{n \rightarrow \infty} f(t+n \omega)$ uniformly on $\mathbb{R}^{+}$, then $f \in A P_{\omega}\left(\mathbb{R}^{+}, \mathbb{X}\right)$.

Let $p \in\left[1, \infty\left[\right.\right.$. The space $B S^{p}\left(\mathbb{R}^{+}, \mathbb{X}\right)$ of all Stepanov bounded functions, with the exponent $p$, consists of all measurable functions $f: \mathbb{R}^{+} \rightarrow \mathbb{X}$ such that $f^{b} \in \mathbb{L}^{\infty}\left(\mathbb{R}, L^{p}([0,1] ; \mathbb{X})\right)$, where $f^{b}$ is the Bochner transform of $f$ defined by $f^{b}(t, s):=f(t+s), t \in \mathbb{R}^{+}, s \in[0,1] . B S^{p}\left(\mathbb{R}^{+}, X\right)$ is a Banach space with the norm

$$
\|f\|_{S^{\mathrm{p}}}=\left\|f^{b}\right\|_{\mathbb{L}^{\infty}\left(\mathbb{R}^{+}, L^{\mathrm{p}}\right)}=\sup _{t \in \mathbb{R}^{+}}\left(\int_{t}^{t+1}\|f(\tau)\|^{p} d \tau\right) .
$$

It is obvious that $L^{p}(\mathbb{R}, \mathbb{X}) \subset B S^{p}(\mathbb{R}, \mathbb{X}) \subset L_{\text {loc }}^{p}(\mathbb{R}, \mathbb{X})$ and $B S^{p}(\mathbb{R}, \mathbb{X}) \subset B S^{q}(\mathbb{R}, \mathbb{X})$ for $p \geq q \geq 1$. We denote by $B S_{0}^{p}\left(\mathbb{R}^{+}, \mathbb{X}\right)$ the subspace of $B S^{p}\left(\mathbb{R}^{+}, \mathbb{X}\right)$ consisting of functions $f$ such that $\int_{t}^{t+1}\|f(s)\|^{p} d s \rightarrow 0$ when $t \rightarrow \infty$.

Define the subspaces of $B S^{p}\left(\mathbb{R}^{+} \mathbb{X}\right)$ by

$$
S^{p} P_{\omega}\left(\mathbb{R}^{+}, X\right)=\left\{f \in B S^{p}\left(\mathbb{R}^{+}, \mathbb{X}\right): \int_{t}^{t+1}\|f(s+\omega)-f(s)\|^{p} d s=0, t \in \mathbb{R}^{+}\right\}
$$


and $B S_{0}^{p}\left(\mathbb{R}^{+}, \mathbb{X}\right)=\left\{f \in B S^{p}\left(\mathbb{R}^{+}, \mathbb{X}\right): \lim _{t \rightarrow \infty} \int_{t}^{t+1}\|f(s)\|^{p} d s=0\right\}$.

Definition 2.3. (see [9]) A function $f \in B S^{p}\left(\mathbb{R}^{+}, \mathbb{X}\right)$ is called asymptotically $\omega$-periodic in the Stepanov sense if it can be expressed as $f=g+h$, where $g \in S^{p} P_{\omega}\left(\mathbb{R}^{+}, \mathbb{X}\right)$ and $h \in B S_{0}^{p}\left(\mathbb{R}^{+}, \mathbb{X}\right)$. The collection of such functions will be denoted by $S^{p} A P_{\omega}\left(\mathbb{R}^{+}, \mathbb{X}\right)$.

Remark 2.2. $A P_{\omega}\left(\mathbb{R}^{+}, \mathbb{X}\right) \subset P_{\omega} L\left(\mathbb{R}^{+}, \mathbb{X}\right) \subset S^{p} P_{\omega}\left(\mathbb{R}^{+}, X\right)$

Theorem 2.5. Let $\Phi: \mathbb{X} \rightarrow \mathbb{Y}$ be a function wich is uniformly continous on the bounded subsets of $\mathbb{X}$ and such that $\Phi$ maps bounded subsets of $\mathbb{X}$ into bounded subsets of $\mathbb{Y}$. Then for all $f \in P_{\omega} L\left(\mathbb{R}^{+}, \mathbb{X}\right)$, the composition theorem $\Phi \circ f:=[t \rightarrow \Phi(f(t))] \in P_{\omega} L\left(\mathbb{R}^{+}, \mathbb{Y}\right)$.

Proof. Since the range of $f$ is bounded, we deduce that $\Phi(f(\cdot))$ is bounded. Let $\epsilon>0$. Then there exists $\delta>0$ such that $\|\Phi(x)-\Phi(y)\|<\epsilon$ for all $x, y \in f\left(\mathbb{R}^{+}\right)$with $\|x-y\| \leq \delta$. Since $\delta>0$, there exists $N=N(\delta) \in \mathbb{N}$ such that if $n \geq N,\|f(t+n \omega)-g(t)\|<\delta$ for each $t \in \mathbb{R}^{+}$. Therefore, when $n \geq N$, $\| \Phi(f(t+n \omega)-\Phi(g(t)) \|<\epsilon$.

Proposition 2.6. Let $(\mathbb{X},\|\cdot\|)$ be a banach space over the field $\mathbb{K}$ where $\mathbb{K}=\mathbb{R}$ or $\mathbb{C}$. If $a(t) \in P L_{\omega}\left(\mathbb{R}^{+}, \mathbb{R}\right)$ and $f(t) \in P L_{\omega}\left(\mathbb{R}^{+}, \mathbb{X}\right)$, then $a(t) f(t) \in$ $P L_{\omega}\left(\mathbb{R}^{+}, \mathbb{X}\right)$.

Proof. We have $\lim _{n \rightarrow \infty} a(t+n \omega)=b(t)$ and $\lim _{n \rightarrow \infty} f(t+n \omega)=g(t)$ for all $t \geq 0$. Since $a(t)$ and $f(t)$ are bounded, there exists $M_{1}, M_{2} \in \mathbb{R}^{+}$such that $|a(t)| \leq M_{1}$ and $\|f(t)\| \leq M_{2}$, for all $t \geq 0$. Since

$\|a(t+n \omega) f(t+n \omega)-b(t) g(t)\| \leq\|a(t+n \omega)-b(t)\| M_{1}+\|f(t+n \omega)-g(t)\| M_{2}$,

we deduce that $\lim _{n \rightarrow \infty} a(t+n \omega) f(t+n \omega)=b(t) g(t)$.

Definition 2.4. A jointly continuous function $f: \mathbb{R}^{+} \times \mathbb{X} \rightarrow \mathbb{X}$ is $\omega$ periodic limit in $t \in \mathbb{R}^{+}$uniformly for $x$ in bounded subsets of $\mathbb{X}$ if for every bounded subset $K$ of $\mathbb{X},\left\{f(t, x): t \in \mathbb{R}^{+}, x \in K\right\}$ is bounded and $\lim _{n \rightarrow \infty} f(t+$ $n \omega, x)=g(t, x)$ exists for each $t \in \mathbb{R}^{+}$and each $x \in K$. The collection of such functions will be denoted by $P_{\omega} L\left(\mathbb{R}^{+} \times \mathbb{X}, \mathbb{X}\right)$. 
Theorem 2.7. If $f: \mathbb{R}^{+} \times \mathbb{X} \rightarrow \mathbb{X}$ is $\omega$-periodic limit in $t \in \mathbb{R}^{+}$uniformly for $x$ in bounded subsets of $\mathbb{X}$ and if $f$ satisfies a Lipschitz condition in $x$ uniformly in $t \in \mathbb{R}^{+}$, then $g$ satisfies the same Lipschitz condition in $x$ uniformly in $t$.

Proof. $f$ satisfies a Lipschitz condition in $x$ uniformly in $t \in \mathbb{R}^{+}$:

$$
\|f(t, x)-f(t, y)\| \leq L\|x-y\|,
$$

for all $x, y \in \mathbb{X}$ and $t \in \mathbb{R}^{+}$, where $L$ is a positive constant. let $\epsilon>0$. Since $f: \mathbb{R}^{+} \times \mathbb{X} \rightarrow \mathbb{X}$ is $\omega$-periodic limit in $t \in \mathbb{R}^{+}$uniformly for $x$ in bounded subsets of $\mathbb{X}$ then there exists $N \in \mathbb{N}$ such that

$$
\|f(t+n \omega, x)-g(t, x)\|<\frac{\epsilon}{2}
$$

and

$$
\|f(t+n \omega, y)-g(t, y)\|<\frac{\epsilon}{2}
$$

when $n \geq N$ for each $t \in \mathbb{R}^{+}$. Observing that

$$
\begin{aligned}
\|g(t, x)-g(t, y)\| & \leq\|g(t, x)-f(t+n \omega, x)\| \\
& +\|f(t+n \omega, x)-f(t+n \omega, y)\| \\
& +\|f(t+n \omega, y)-g(t, y)\|,
\end{aligned}
$$

we deduce so that

$$
\|g(t, x)-g(t, y)\|<\epsilon+L\|x-y\|
$$

when $n \geq N$. Since $\epsilon$ is arbitrary, we can write

$$
\|g(t, x)-g(t, y)\| \leq L\|x-y\| .
$$

Theorem 2.8. (see [10]) Let $f: \mathbb{R}^{+} \times \mathbb{X} \rightarrow \mathbb{X}$ be $\omega$-periodic limit on $t \in \mathbb{R}^{+}$uniformly for $x$ in bounded subsets of $\mathbb{X}$ and assume that $f$ satisfies a Lipschitz condition in $x$ uniformly in $t \in \mathbb{R}^{+}$:

$$
\|f(t, x)-f(t, y)\| \leq L\|x-y\|,
$$

for all $x, y \in \mathbb{X}$ and $t \in \mathbb{R}^{+}$, where $L$ is a positive constant. Let $\varphi: \mathbb{R}^{+} \rightarrow \mathbb{X}$ be $\omega$-periodic limit. Then the function $F: \mathbb{R}^{+} \rightarrow \mathbb{X}$ defined by $F(t)=f(t, \varphi(t))$ is $\omega$-periodic limit. 


\section{Main Results}

Now we make the following hypthesis:

(H1) : The function $f: \mathbb{R}^{+} \times \mathbb{X} \rightarrow \mathbb{X}$ is $\omega$-periodic limit in $t \in \mathbb{R}^{+}$uniformly for $x$ in bounded subsets of $\mathbb{X}$ and $f$ satisfies a Lipschitz condition in $x$ uniformly in $t \in \mathbb{R}^{+}$:

$$
\|f(t, x)-f(t, y)\| \leq L\|x-y\|,
$$

for all $x, y \in \mathbb{X}$ and $t \in \mathbb{R}^{+}$, where $L$ is a positive constant.

(H2): $A(t)$ generates an exponentially stable evolutionnary process $(U(t, s))_{t \geq s}$ in $\mathbb{X}$, that is, a two-parameter family of bounded linear operators that satisfies the following conditions:

1. $U(t, t)=I$ for all $t \in \mathbb{R}$ where $I$ is the identity operator.

2. $U(t, s) U(s, r)=U(t, r)$ for all $t \geq s \geq r$.

3. The map $(t, s) \mapsto U(t, s) x$ is continuous for every fixed $x \in \mathbb{X}$.

4. $U(t+\omega, s+\omega)=U(t, s)$ for all $t \geq s(\omega$-periodicity $)$.

5. There exist $K>0$ and $a>0$ such that $\|U(t, s)\| \leq K e^{-\delta(t-s)}$ for $t \geq s$.

Definition 3.1. We assume (H1) is satisfied and that $A(t)$ generates an evolutionary process $(U(t, s))_{t \geq s}$ in $\mathbb{X}$. The continuous function $x$ given by

$$
x(t)=U(t, 0) c_{0}+\int_{0}^{t} U(t, s) f(s, x(s)) d s
$$

is called the mild solution of equation (1).

Lemma 3.1. We assume that (H2) is satisfied and that $f \in P_{\omega} L\left(\mathbb{R}^{+}, \mathbb{X}\right)$. Then

$$
(\wedge f)(t)=\int_{0}^{t} U(t, s) f(s) d s \in A P_{\omega}\left(\mathbb{R}^{+}, \mathbb{X}\right), t \in \mathbb{R}^{+} .
$$

Proof. We put

$$
v(t)=\int_{0}^{t} U(t, s) f(s) d s .
$$

Since $f \in P_{\omega} L\left(\mathbb{R}^{+}, \mathbb{X}\right)$,

$$
\lim _{n \rightarrow \infty} f(t+n \omega)=g(t)
$$


is well defined for each $t \in \mathbb{R}^{+}$. There exists also a positive constant $K$ such that $\|g\|_{\infty} \leq\|f\|_{\infty} \leq K$ and $g(t)=g(t+\omega)$. We observe that

$$
\begin{aligned}
v(t+n \omega) & =\int_{0}^{t+n \omega} U(t+n \omega, s) f(s) d s \\
& =\int_{-n \omega}^{t} U(t+n \omega, s+n \omega) f(s+n \omega) d s \\
& =\int_{-n \omega}^{t} U(t, s) f(s+n \omega) d s \\
& =\int_{-n \omega}^{0} U(t, s) f(s+n \omega) d s+\int_{0}^{t} U(t, s) f(s+n \omega) d s \\
& =I_{1}(t, n)+I_{2}(t, n)
\end{aligned}
$$

Next we will prove that $I_{1}(t, n)$ is a Cauchy sequence in $\mathbb{X}$ for each $t \in \mathbb{R}^{+}$. Let $\epsilon>0$. For any $p \in \mathbb{N}, n \in \mathbb{N}$, we observe that

$$
\begin{aligned}
I_{1}(t, n+p)-I_{1}(t, n) & =\int_{-(n+p) \omega}^{0} U(t, s) f(s+(n+p) \omega) d s \\
& -\int_{-n \omega}^{0} U(t, s) f(s+n \omega) d s \\
& =\int_{-(n+p) \omega}^{-n \omega} U(t, s) f(s+(n+p) \omega) d s \\
& +\int_{-n \omega}^{0} U(t, s)(f(s+(n+p) \omega)-f(s+n \omega)) d s \\
& =I_{3}(t, n, p)+I_{4}(t, n, p) .
\end{aligned}
$$

Consider the term $I_{3}(t, n, p)$.

$$
\begin{aligned}
\left\|I_{3}(t, n, p)\right\| & \leq \int_{-(n+p) \omega}^{-n \omega}\|U(t, s)\|\|f(s+(n+p) \omega)\| d s \\
& \leq \int_{-(n+p) \omega}^{-n \omega} M e^{-\delta(t-s)} K d s \\
& \leq \int_{n \omega}^{(n+p) \omega} M K e^{-\delta(t+s)} d s \\
& \leq K M \int_{n \omega}^{\infty} e^{-\delta(t+s)} d s
\end{aligned}
$$




$$
\leq \frac{K M}{\delta} e^{-\delta n \omega}
$$

We can choose $N_{1} \in \mathbb{N}$ such that $\frac{K M}{\delta} e^{-\delta n \omega} \leq \epsilon$ when $n \geq N_{1}$. Therefore $\left\|I_{3}(t, n, p)\right\| \leq \epsilon$ whenever $n \geq N_{1}$ uniformly for $t \in \mathbb{R}^{+}$.

For $n \geq N_{1}$, consider $I_{4}(t, n, p)$.

$$
\begin{aligned}
I_{4}(t, n, p) & =\int_{-N_{1} \omega}^{0} U(t, s)(f(s+(n+p) \omega)-f(s+n \omega)) d s \\
& +\int_{-n \omega}^{-N_{1} \omega} U(t, s)(f(s+(n+p) \omega)-f(s+n \omega)) d s \\
& =I_{5}(t, n, p)+I_{6}(t, n, p) .
\end{aligned}
$$

We have

$$
\begin{aligned}
& \left\|I_{5}(t, n, p)\right\| \leq \int_{-N_{1} \omega}^{0}\|U(t, s)\| \|(f(s+(n+p) \omega)-f(s+n \omega) \| d s \\
& \leq \int_{-N_{1} \omega}^{0} M e^{-\delta(t-s)} \|(f(s+(n+p) \omega)-f(s+n \omega) \| d s \\
& \leq \int_{0}^{N_{1} \omega} M e^{-\delta(t+s)} \|(f((n+p) \omega-s)-f(n \omega-s) \| d s \\
& \leq \int_{0}^{N_{1} \omega} M e^{-\delta\left(t+N_{1} \omega-s\right)} \|\left(f\left(\left(n-N_{1}+p\right) \omega+s\right)\right. \\
& \text { - } \quad f\left(\left(n-N_{1}\right) \omega+s\right) \| d s \\
& \leq \int_{0}^{N_{1} \omega} M e^{-\delta\left(N_{1} \omega-s\right)} \|\left(f\left(\left(n-N_{1}+p\right) \omega+s\right)\right. \\
& \text { - } f\left(\left(n-N_{1}\right) \omega+s\right) \| d s \\
& \leq \int_{0}^{N_{1} \omega} M e^{-\delta\left(N_{1} \omega-s\right)} \|\left(f\left(\left(n-N_{1}+p\right) \omega+s\right)-g(s) \| d s\right. \\
& +\int_{0}^{N_{1} \omega} M e^{-\delta\left(N_{1} \omega-s\right)} \|\left(f\left(\left(n-N_{1}\right) \omega+s\right)-g(s) \| d s .\right.
\end{aligned}
$$

For each $s \in\left[0, N_{1} \omega\right]$, we have

$$
M e^{-\delta\left(N_{1} \omega-s\right)} \|\left(f\left(\left(n-N_{1}+p\right) \omega+s\right)-g(s) \| \leq 2 M K e^{-\delta\left(N_{1} \omega-s\right)}\right.
$$

and

$$
\int_{0}^{N_{1} \omega} 2 M K e^{-\delta\left(N_{1} \omega-s\right)} d s=\frac{2 M K}{\delta}\left(1-e^{-\delta N_{1} \omega}\right) .
$$


Since $f \in P_{\omega} L\left(\mathbb{R}^{+}, \mathbb{X}\right)$, for each $s \in\left[0, N_{1} \omega\right]$, we have

$$
\lim _{n \rightarrow \infty} M e^{-\delta\left(N_{1} \omega-s\right)} \|\left(f\left(\left(n-N_{1}+p\right) \omega+s\right)-g(s) \|=0 .\right.
$$

By the Lebesgue's Dominated Convergence Theorem, we deduce that

$$
\lim _{n \rightarrow \infty} \int_{0}^{N_{1} \omega} M e^{-\delta\left(N_{1} \omega-s\right)} \|\left(f\left(\left(n-N_{1}+p\right) \omega+s\right)-g(s) \| d s=0 .\right.
$$

Similarly, we have

$$
\lim _{n \rightarrow \infty} \int_{0}^{N_{1} \omega} M e^{-\delta\left(N_{1} \omega-s\right)} \|\left(f\left(\left(n-N_{1}\right) \omega+s\right)-g(s) \| d s=0 .\right.
$$

Therefore, we can select $N_{2} \in \mathbb{N}\left(N_{2}>N_{1}\right)$ such that $\left\|I_{5}(t, n, p)\right\| \leq \epsilon$ whenever $n \geq N$ uniformly for $t \in \mathbb{R}^{+}$.

Now we consider the term $I_{6}(t, n, p)$.

$$
\begin{aligned}
\left\|I_{6}(t, n, p)\right\| & \leq \int_{-n \omega}^{-N_{1} \omega}\|U(t, s)\|\|f(s+(n+p) \omega)-f(s+n \omega)\| d s \\
& \leq 2 K M \int_{-n \omega}^{-N_{1} \omega} e^{-\delta(t-s)} d s \\
& \leq 2 K M \int_{N_{1} \omega}^{n \omega} e^{-\delta(t+s)} d s \\
& \leq 2 K M \int_{N_{1} \omega}^{\infty} e^{-\delta(t+s)} d s \\
& \leq \frac{2 K M}{\delta} e^{-\delta N_{1} \omega} \\
& \leq 2 \epsilon
\end{aligned}
$$

uniformly for $t \in \mathbb{R}^{+}$. Since

$$
\left\|I_{1}(t, n+p)-I_{1}(t, n)\right\| \leq\left\|I_{3}(t, n, p)\right\|+\left\|I_{5}(t, n, p)\right\|+\left\|I_{6}(t, n, p)\right\|
$$

we deduce that $\left\|I_{1}(t, n+p)-I_{1}(t, n)\right\| \leq 4 \epsilon$ when $n \geq N_{2}$. Therefore $I_{1}(t, n)$ is a cauchy sequence. So we can denote $h(t)=\lim _{n \rightarrow \infty} I_{1}(t, n)$ for each $t \in \mathbb{R}^{+}$. Note also that $h(t)=\lim _{n \rightarrow \infty} I_{1}(t, n)$ uniformly for $t \in \mathbb{R}^{+}$.

Now consider the term $I_{2}(t, n)$. Since $g$ is measurable, $\int_{0}^{t} U(t, s) g(s) d s$ is well defined for each $t \in \mathbb{R}^{+}$. For $m \omega \leq t<(m+1) \omega, m \in \mathbb{N}$, we have

$$
\left\|I_{2}(t, n)-\int_{0}^{t} U(t, s) g(s) d s\right\| \leq \int_{0}^{t}\|U(t, s)\|\|f(s+n \omega)-g(s)\| d s
$$




$$
\begin{aligned}
& \leq \int_{0}^{t} M e^{-\delta(t-s)}\|f(s+n \omega)-g(s)\| d s \\
& \leq \int_{0}^{m \omega} M e^{-\delta(t-s)}\|f(s+n \omega)-g(s)\| d s \\
& +\int_{m \omega}^{t} M e^{-\delta(t-s)}\|f(s+n \omega)-g(s)\| d s \\
& \leq M \sum_{k=0}^{m-1} \int_{k \omega}^{(k+1) \omega} e^{-\delta(t-s)}\|f(s+n \omega)-g(s)\| d s \\
& +M \int_{m \omega}^{(m+1) \omega} e^{-\delta(t-s)}\|f(s+n \omega)-g(s)\| d s \\
& \leq M \sum_{k=0}^{m-1} e^{-\delta(t-(k+1) \omega)} \int_{k \omega}^{(k+1) \omega}\|f(s+n \omega)-g(s)\| d s \\
& +M \int_{m \omega}^{(m+1) \omega}\|f(s+n \omega)-g(s)\| d s
\end{aligned}
$$

For each $s \in[0, \omega]$, we have $\lim _{n \rightarrow \infty}\|f(s+n \omega)-g(s)\|=0$ and $\|f(s+n \omega)-g(s)\| \leq$ $2 K$. By Lebesgue's Dominated Convergence theorem, we obtain

$$
\lim _{n \rightarrow \infty} \int_{0}^{\omega}\|f(s+n \omega)-g(s)\|=0 .
$$

For $\epsilon>0$, there existe $N_{3} \in \mathbb{N}$ such that

$$
\int_{0}^{\omega}\|f(s+n \omega)-g(s)\| \leq \epsilon
$$

when $n \geq N_{3}$. For any $i \in \mathbb{N}$, we have

$$
\begin{aligned}
\int_{i \omega}^{(i+1) \omega}\|f(s+n \omega)-g(s)\| d s & =\int_{0}^{\omega}\|f(s+i \omega+n \omega)-g(s+i \omega)\| d s \\
& =\int_{0}^{\omega}\|f(s+i \omega+n \omega)-g(s)\| d s \\
& \leq \epsilon
\end{aligned}
$$

when $n \geq N_{3}$. Therefore

$$
\left\|I_{2}(t, n)-\int_{0}^{t} U(t, s) g(s) d s\right\| \leq M \sum_{k=0}^{m-1} e^{-\delta(t-(k+1) \omega)} \epsilon+M \epsilon
$$




$$
\leq\left(\frac{1}{1-e^{-\delta \omega}}+1\right) M \epsilon
$$

when $n \geq N_{3}$ uniformly for $t \in \mathbb{R}^{+}$. Therefore

$$
\lim _{n \rightarrow \infty} I_{2}(t, n)=\int_{0}^{t} U(t, s) g(s) d s
$$

uniformly for $t \in \mathbb{R}^{+}$. Now we have $\lim _{n \rightarrow \infty} v(t+n \omega)=\lim _{n \rightarrow \infty} I_{1}(t, n)+$ $\lim _{n \rightarrow \infty} I_{2}(t, n)=h(t)+\int_{0}^{t} U(t, s) g(s) d s$ uniformly for $t \in \mathbb{R}^{+}$. We deduce that $v \in A P_{\omega}\left(\mathbb{R}^{+}, \mathbb{X}\right)$.

Theorem 3.2. We assume that the hypothesis $(\mathbf{H 1})$ and $(\mathbf{H 2})$ are satisfied. Then (1) has a unique Asymptotically $\omega$-periodic mild solution provided

$$
\Theta:=\frac{L M}{a}<1
$$

Proof. We define the nonlinear operator $\Gamma$ by the expression

$$
\begin{aligned}
(\Gamma \phi)(t) & =U(t, 0) c_{0}+\int_{0}^{t} U(t, s) f(s, \phi(s)) d s \\
& =U(t, 0) c_{0}+\left(\wedge_{1} \phi\right)(t)
\end{aligned}
$$

where

$$
\left(\wedge_{1} \phi\right)(t)=\int_{0}^{t} U(t, s) f(s, \phi(s)) .
$$

According to the hypothesis (H.2), we have

$$
\|U(t, 0)\| \leq M e^{-a t}
$$

Therefore $\lim _{t \rightarrow \infty}\|U(t, 0)\|=0$.

According to the Theorem 2.8, the function $t \rightarrow f(t, u(t))$ belongs to $P_{\omega} L\left(\mathbb{R}_{+}, \mathbb{X}\right)$. According to the Lemma 3.1 the operator $\wedge_{1}$ maps $A P_{\omega}\left(\mathbb{R}^{+}, \mathbb{X}\right)$ into itself. Therefore the operator $\Gamma$ maps $A P_{\omega}\left(\mathbb{R}^{+}, \mathbb{X}\right)$ into itself.

We have

$$
\begin{aligned}
\|(\Gamma \phi)(t)-\Gamma \psi)(t) \| & =\left\|\int_{0}^{t} U(t, s)(f(s, \phi(s))-f(s, \psi(s))) d s\right\| \\
& \leq \int_{0}^{t}\|U(t, s)\|\|f(s, \phi(s))-f(s, \psi(s))\| d s \\
& \leq L \int_{0}^{t}\|U(t, s)\|\|\phi(s)-\psi(s)\| d s
\end{aligned}
$$




$$
\begin{aligned}
& \leq L M \int_{0}^{t} e^{-a(t-s)}\|\phi(s)-\psi(s)\| d s \\
& \leq L M \int_{0}^{t} e^{-a(t-s)}\|\phi-\psi\|_{\infty} d s \\
& \leq L M\left(\frac{1-e^{-a t}}{a}\right)\|\phi-\psi\|_{\infty} \\
& \leq \frac{L M}{a}\|\phi-\psi\|_{\infty} .
\end{aligned}
$$

Hence we have :

$$
\|\Gamma \phi-\Gamma \psi\|_{\infty} \leq \frac{L M}{a}\|\phi-\psi\|_{\infty}
$$

which proves that $\Gamma$ is a contraction and we conclude that $\Gamma$ has a unique fixed point in $A P_{\omega}\left(\mathbb{R}^{+}, \mathbb{X}\right)$. The proof is complete.

\section{References}

[1] C. Cuevas and J.C. de Souza. S asymptotically $\boldsymbol{\omega}$-periodic solutions of semilinear fractional integro-differential equations. Appl. Math. Lett, 22, 2009, 865-870, DOI: 10.1016/j.aml.2008.0\%.013.

[2] C. Cuevas and C. Lizanna. S asymptotically $\boldsymbol{\omega}$-periodic solutions of semilinear Volterra equations. Math. Meth. Appl. Sci, 33, 2010, 1628-1636, DOI: 10.1002/mma.1284.

[3] C. Cuevas and C. Lizanna. Existence of $\mathbf{S}$ asymptotically $\boldsymbol{\omega}$-periodic solutions for twotimes fractional order differential equations. Southeast.Asian Bull.Math. 37, 2013, 683690, DOI: 10.1016/j.na.2009.09.00.

[4] W. Dimbour J-C. Mado. S asymptotically $\omega$-periodic solution for a nonlinear differential equation with piecewise constant argument in a Banach space. CUBO A Mathematical Journal 16(13), 2014, 55-65,DOI: 10.4067/S0719-06462014000300005.

[5] W. Dimbour, G.Mophou and G.M. N'Guérékata. S asymptotically w-periodic solution for partial differential equations with finite delay. Electron.J.Differ.Equa. 117, 2011, 1-12.

[6] H. R. Henríquez Asymptotically periodic solutions of abstract differential equations. Nonlinear Analysis, 80 (2013), DOI: 10.1016/j.na.2012.10.010.

[7] H. R. Henríquez, M. Pierri and P. Táboas. On S asymptotically $\boldsymbol{\omega}$-periodic function on Banach spaces and applications. J. Math. Anal Appl. 343, 2008, 1119-1130, DOI: 10.1016/j.jmaa.2008.02.023.

[8] S. Nicola, M. Pierri. A note on S-asymptotically periodic functions. Nonlinear Anaklysis, Real World Application, 10 (2009), DOI: 10.1016/j.nonrwa.2008.09.011.

[9] R. Xie and C. Zhang. Criteria of asymptotic $\omega$-periodicity and their applications in a class of fractional differential equations. Advances in Difference Equations, 1, 2015, DOI: 10.1186/s13662-015-0404-z.

[10] R. Xie and C. Zhang. Space of $\omega$-Periodic Limit Functions and its Applications to an Abstract Cauchy Problem. Journal of function Spaces, 2015, DOI: 10.1155/2015/953540. 
[11] Z.Xia. Asymptotically periodic solutions of semilinear fractional integro-differential equations. Advances in Difference Equations, 1, 2014, 1-19, DOI: 10.1186/1687-1847-2014-9

[12] Zhong-Hua Wu. Asymptotic periodicity for a class of fractional integro-differential equations. J. Nonlinear Sci. Appl. 9, 2016, 506-517. 
